\title{
Theory of Structural Glasses and Supercooled Liquids
}

\author{
Vassiliy Lubchenko \\ Department of Chemistry, University of Houston, Houston, TX 77204-5003 \\ Peter G. Wolynes \\ Department of Chemistry and Biochemistry and Department of Physics, \\ University of California at San Diego, La Jolla, CA 92093-0371
}

(Dated: September 21, 2018)

\begin{abstract}
We review the Random First Order Transition Theory of the glass transition, emphasizing the experimental tests of the theory. Many distinct phenomena are quantitatively predicted or explained by the theory, both above and below the glass transition temperature $T_{g}$. These include: the viscosity catastrophe and heat capacity jump at $T_{g}$, and their connection; the non-exponentiality of relaxations and their correlation with the fragility; dynamic heterogeneity in supercooled liquids owing to the mosaic structure; deviations from the Vogel-Fulcher law, connected with strings or fractral cooperative rearrangements; deviations from the Stokes-Einstein relation close to $T_{g}$; aging, and its correlation with fragility; the excess density of states at cryogenic temperatures due to two level tunneling systems and the Boson Peak.
\end{abstract}

\section{Contents}

\section{Introduction}

\section{Basic Phenomenology of the Structural Glass Transition}

\section{Classical Theory of the Glass Transition and Supercooled Liquids \\ A. Emergence of a Free Energy Landscape of Aperiodic Structural States \\ B. The Library of Local States \\ C. Activated Motions between Local States \\ D. Dynamics near the Crossover \\ E. Aging}

\section{Quantum Theory of Glasses}

\section{Concluding Remarks - Future Directions}

References

\section{INTRODUCTION}

School children have their earliest exposure to the subject of physical chemistry when they hear about "the states of matter." They are taught there are gases, liquids, and solids. Van der Waals revealed to scientists that gases and liquids differed actually only quantitatively [1]. The rigidity of solids, which defines them macroscopically, on the other hand, has been usually traced to their qualitatively different, periodic ordered structure [2] - an idea that already occurred to Kepler [3]. This idea is correct for crystals. The existence of structural glasses, i.e. amorphous substances that are rigid, calls this understanding into question, however. The utility and adaptability of glasses arises from the way their properties depend on their preparation history and their seeming con- tinuity with the supercooled liquid state. In contrast to dilute gases and crystalline solids, where the properties can be directly inferred from the intermolecular forces, liquids have generally enjoyed a reputation of mystery among physical chemists. Until recently, the transition to the "glassy state" was deemed specifically one of the most obscure enigmas by many in the theoretical physics and chemistry communities [4-6]. Despite this reputation, a constructive molecular theory of structural glasses and the dynamics of supercooled liquids has been developed. This theory starts with the intermolecular forces. While it would suffer from all the well-known issues of microscopic modeling, if it were used to predict glass transition temperatures from scratch, for example, the theory does explain extremely well the known (and rather unusual) phenomenology of supercooled liquids and the strange properties of glasses at low temperatures. The theory also does make a large number of quantitative predictions without adjustable parameters that are borne out quite well by experiment. The purpose of this article is to briefly explain the basic ideas of this theory and how its predictions compare with experiment.

The basic idea of the theory of structural glasses is to consider them to be aperiodic crystals. Since Bernal's time at least, it has been appreciated that an aperiodic structure can be mechanically stable [7]. Strictly speaking, Kepler's assumption of periodicity would not be needed for rigidity, were it not for thermal motions. In contrast to periodic crystals, there are extremely many aperiodic structures and it is hard to see why they should have any difficulty interconverting thereby allowing flow. But we know they indeed do have such a difficulty since the flow of glass is nearly imperceptible. It is this fact that a theory of the glass transition must explain. We shall see the theory of structural glasses then generalizes the theory of an ordinary first order freezing transition, which gives a periodic crystalline structure, to a very much analogous change that involves freezing into a set of aperiodic structures which are statistically distributed 


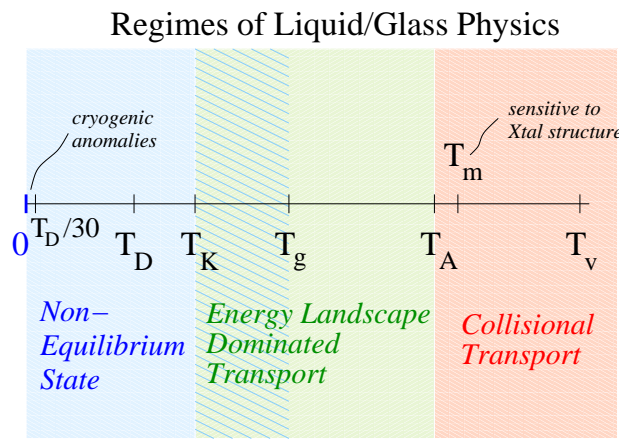

FIG. 1: Regimes of the aperiodic condensed molecular phase are shown, ranging between a dilute gas and a frozen glass. $T_{v}$ is the vaporization temperature, $T_{m}$ the melting point. $T_{A}$ represents the temperature signalling the crossover to activated motions, which is usually but not always below $T_{m} . T_{g}$ is the glass transition temperature which depends on the time scale of measurement. Below $T_{g}$ the system is out of equilibrium and ages. $T_{K}$ is the Kauzmann temperature (see text). $T_{D}$ is the Debye temperature which signals the quantization of vibrational motions. Below $T_{D} / 30$, or so, the thermal properties of the system can be phenomenologically described as arising from a collection of two level systems. Just above this point, additional quantum excitations, sometimes called the Boson peak, are present.

in energy. We call this change a "random first order transition."

We will begin this review by discussing a small number of key experimental signatures of the glass transition in Section II. In Section III, we construct the microscopic picture of the glassy state and the transition to it from a supercooled liquid, following the random first order transition theory. A variety of temperatures characterizes glasses and liquids in this theory. They are graphically summarized in Fig.1. We will define these scales more precisely in the discussion below and we recommend the reader to often refer to this figure. Starting with a onecomponent gas, one may cool it down and compress it until it condenses below the critical point, $T_{v}$, usually above the crystallization temperature $T_{m}$. In this temperature range, an effective description in terms of collisional transport is valid: a liquid is just a very dense gas held together by an average attractive force. No two molecules are likely to reside near each other for any significant time. The time scales for molecular permutations and collisions are comparable in this regime. All the pertinent information about particle-particle interactions may be encoded in low order correlation functions that may be computed or extracted experimentally from scattering experiments. In a supercooled liquid, on the other hand, molecules maintain their immediate set of neighbors for hundreds of collisional or vibrational periods. This occurs near the temperature $T_{A}$. These local spatial patterns persist ever longer as the temperature is lowered. Interconversion between such structures occurs both above and below the glass transition temperature $T_{g}$, which depends on the preparation time scale. The in-

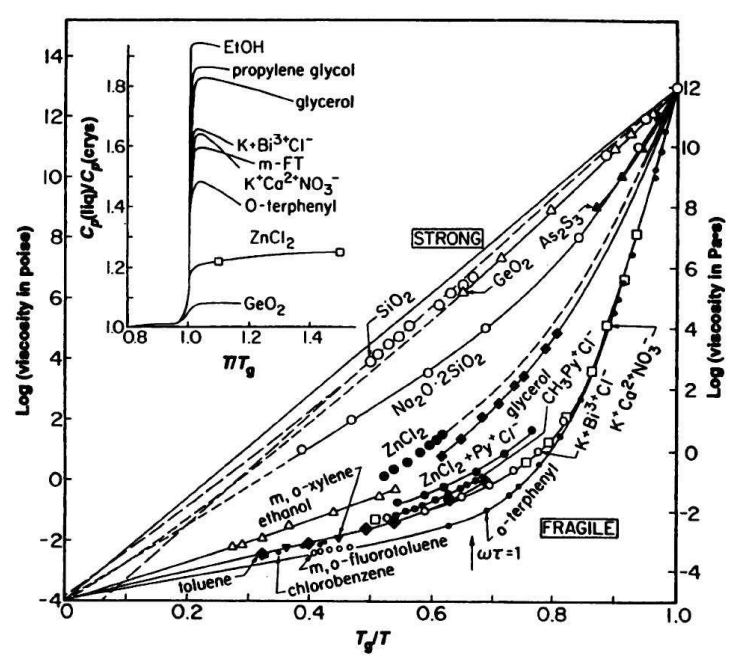

FIG. 2: The viscosities of several supercooled liquids are plotted as functions of the inverse temperature. Substances with almost-Arrhenius-like dependences are said to be strong liquids, while the visibly convex curves are described as "fragile" substances. The full dynamic range from about a picosecond, on the lower viscosity side, to $10^{4}$ seconds or so when the viscosity reaches to $10^{13}$ poise. This figure is taken from Ref.[6].

terconversion is called the $\alpha$-relaxation when the material remains in equilibrium. However, when $\alpha$-relaxation becomes too slow and only a fraction of the interconversions have time to occur, the material is a glass that "ages". Even at cryogenic temperatures (liquid He and below), a certain fraction of the sample will harbor several kinetically accessible states. Interconversions can still occur by tunneling. These quantum motions are discussed in Section IV. In the final Section V, we make concluding remarks and highlight some open questions in the field.

\section{BASIC PHENOMENOLOGY OF THE STRUCTURAL GLASS TRANSITION}

Liquids exhibit a remarkable range of dynamical behaviors within a relatively narrow temperature interval. Viscosity, for example, varies over a tremendous dynamic range: Fig.2 reproduces the celebrated "Angell" plot of the viscosities for superooled liquids as functions of the inverse temperature scaled to their respective glass transition temperatures, where the relaxation time is roughly one hour [6]. The temperature dependence of other structural relaxation times, such as the inverse of the lowest frequency peak of the dielectric susceptibility, follow a similar temperature dependence and can be described by the so-called Vogel-Fulcher (VF) law, to a first approximation:

$$
\tau=\tau_{0} e^{D T_{0} /\left(T-T_{0}\right)},
$$

where the material coefficient $D$ is called the liquid's "fragility". The Vogel-Fulcher fits work better in the 


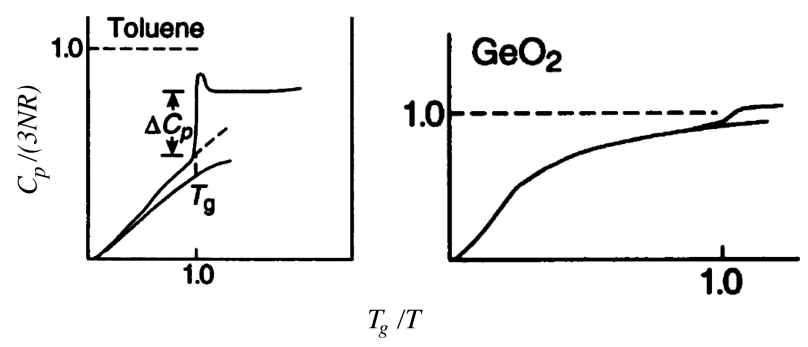

FIG. 3: Shown are the temperature dependences of the heat capacity for two substances, both for the glassy and crystalline counterparts. This figure is a fragment of Fig.2 from Ref.[6].

vicinity of the temperature $T_{0}$, which is also a material dependent quantity. Near $T_{0}$, the relaxation times grow most rapidly and would appear to diverge, if one had the patience to equilibrate the liquid for cosmological times. Deviations from the VF law occur, of course, when the substance falls out of equilibrium near the laboratory $T_{g}$.

In parallel with the dynamical changes upon supercooling, there are apparent thermodynamic changes. When the system falls out of equilibrium, thermodynamic susceptibilities nearly discontinuously decrease. The most interesting of these is the heat capacity shown in Fig.3. The heat capacity allows one to monitor the entropy of liquid configurations of a supercooled fluid. This discontinuity in the heat capacity is well approximated by subtracting from the measured heat capacity of the liquid the heat capacity of the corresponding crystal. By integrating in temperature, one obtains the part of the entropy that comes from the diversity of liquid configurations, see Fig.4. This excess "configurational" entropy is typically of the order of a few $k_{B}$ per rigid molecular unit (such as a $\mathrm{SiO}_{4}$ tetrahedron in silica, or an aromatic ring in TNB).

When extrapolated below the glass transition temperature $T_{g}$, the configurational entropy, $s_{c}$, would appear to vanish at a temperature $T_{K}[9]$, called the Kauzmann temperature. Given the magnitude of the heat capacity jump $\Delta c_{p}$, at the glass transition, various ways to fit the temperature dependence of the configurational entropy can be proposed, such as [8]:

$$
s_{c}=\Delta c_{p}\left(1-T_{K} / T\right) .
$$

While there have been disputes about precisely how $s_{c}$ should be extrapolated [10], it is fairly clear that the dynamic $T_{0}$ is equal to the thermodynamic $T_{K}$ for all glassformers, see Fig.4. That the missing translational motion is the main contributor to the entropy loss is explicitly confirmed by the frequency dependent heat capacity measurements of Nagel and coworkers [11], which show the process contributing to the bulk calorimetry occurs again exactly at the time scales implied by kinetic measurements, such as the frequency dependent relaxations, mechanical or otherwise. That this is a time scale for microscopic movements is also evidenced by the "plateau" in time-resolved neutron scattering, which confirms the

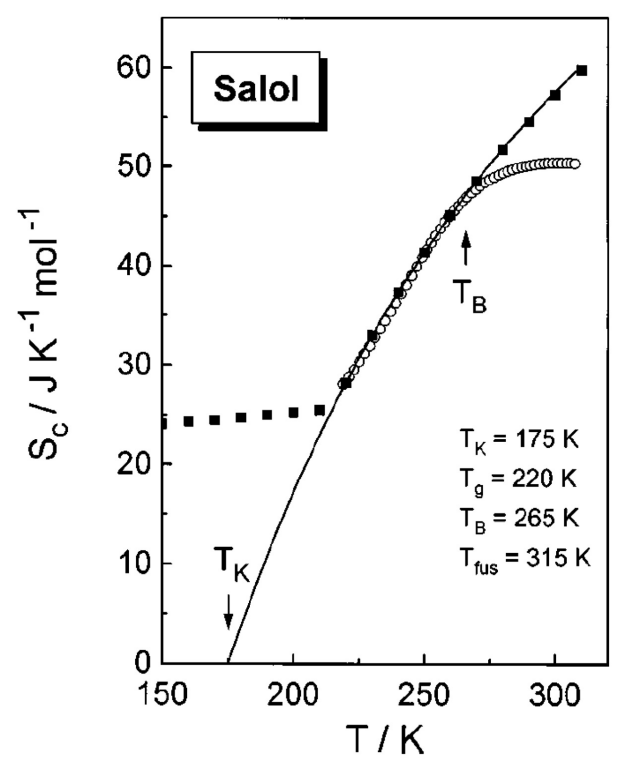

FIG. 4: This figure, from Ref.[8], illustrates the extrapolation of the experimentally determined configurational entropy (shown by squares) below the dynamic glass transition temperature $T_{g}$. This graph illustrates that the Kauzmann temperature $T_{K}$ is equal to the temperature $T_{0}$ from Eq.(1), at which the relaxation times would strictly diverge. The circles represent $1 / \ln (\tau)$, times a convenient scaling constant.

local molecular environment rearranges on the same time scale [12], see also Fig.6.

Rather modest deviations from the consonance of time scales of molecular motions have excited much attentionthese are often discussed as "decoupling." For example, the diffusion coefficient differs from its Stokes-Einstein value by about two orders of magnitude at $T_{g}$. This is two orders of magnitude out of 14 . Leaving aside these modest effects, a staggering amount of data acquired during the past century show the dramatic slowing is essentially shared by nearly all motions.

\section{CLASSICAL THEORY OF THE GLASS TRANSITION AND SUPERCOOLED LIQUIDS}

\section{A. Emergence of a Free Energy Landscape of Aperiodic Structural States}

In gases, molecules spend relatively little time near each other, moving in straight lines separated by collision events, as illustrated in Fig.5(a). In the denser, colder liquid state, the collision duration grows while the time between collisions becomes smaller. These times become comparable in the liquid state. Nevertheless, any two molecules are unlikely to stay together for any significant time. In other words, consecutive collisions near the critical point usually, but not always, occur between different pairs, as in Fig.5(b). The high temperature liquid is just a dense gas, dynamically. 


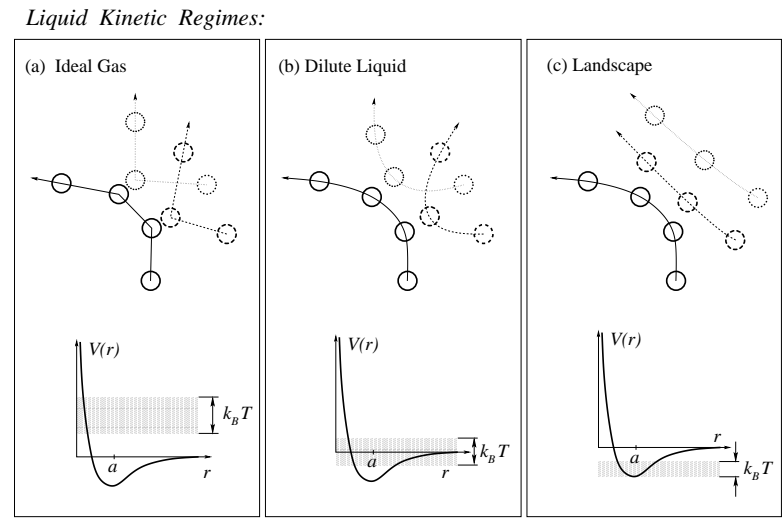

FIG. 5: The three panels demonstrate the three relatively distinct kinetic regimes of an equilibrium liquid, or a conditionally equilibrium liquid, as the case would be below the melting temperature $T_{m}$. See text for explanation. The bottom portions illustrate the region of the two-particle potentials explored in the corresponding regimes.

As temperature decreases and density increases still further, groups of consecutive collisions will occur ever more often within nearly the same set of molecules, see Fig.5(c). Each molecule begins to reside within a specific "cage" for a discernible time. Alternatively, we may say persistent local liquid structures form at these temperatures. Perturbative expansions, that keep track of the very many collision sequences within local regions, require computing increasingly higher order correlation functions [13]. At sufficiently high density, it is convenient to change one's perspective of liquid state dynamics from considering the dynamics of a dense gas to motions within sets of aperiodic crystal structures. One notices that even though each long-living structure involves many correlated many-particle events, each particle within a structure is most often doing something quite simple. It is vibrating about a fixed location and only occasionally moves to a new location. This is an experimental fact, as evidenced by neutron scattering data which exhibit a plateau in the time dependent structure factor, see Fig.6.

The motion of a particle is ever more confined to occur within the cage, so individual molecular bonds distort very little, allowing one to treat these persistent mo- tions as approximately harmonic at high enough densities. When a particle takes up a new position of residence, this implies it has moved beyond a certain threshold distance (see below). This threshold is reminiscent of the Lindemann melting criterion [14] for periodic crystals. When a particle takes up a new residence position it usually does not do so alone. Individual bond breaking and vacancy formation are just as rare processes in a deeply supercooled liquid as they are in crystals, which have comparable local density and stiffness. Instead, usually a group of particles moves whenever a net displacement of an individual molecule occurs.

The mostly harmonic nature of individual displace-

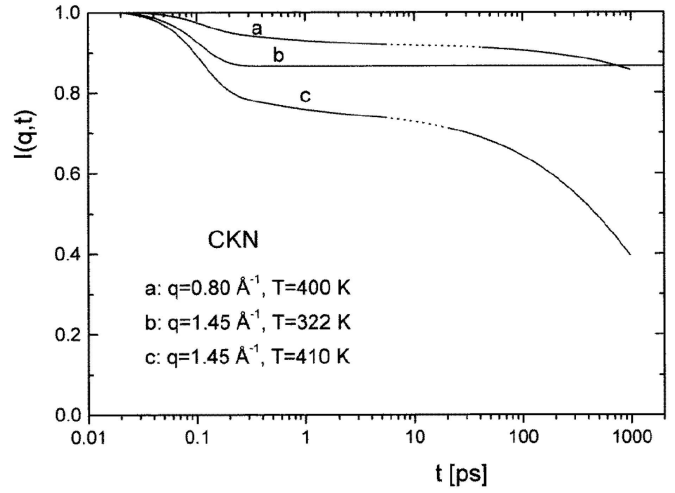

FIG. 6: The plateaus in time resolved neutron scattering structure function provide a direct proof of long-lived local structure in supercooled liquids. The curve (b) was obtained below the glass transition. The very flat plateau implies there is only a relatively small degree of structural rearrangements in the frozen lattice. This figure is taken from Ref.[12]:

ments in long-living structures allows one to use a simple theory to describe the emergence of aperiodic crystals using density functionals $[15,16]$. In equilibrium statistical mechanics the free energy can be written as a functional of a non-uniform density. There is an entropic cost for forming such a non-uniform density, but if particles are localized, they can avoid each other to compensate. By combining the ideal gas localization entropy with an effective interaction, Ramakrishnan and Yussouff [17, 18] wrote:

$$
F[\rho(\mathbf{r})]=k_{B} T \int d^{3} \mathbf{r} \rho(\mathbf{r})[\ln \rho(\mathbf{r})-1]+\frac{1}{2} \iint d^{3} \mathbf{r} d^{3} \mathbf{r}^{\prime}\left[\rho(\mathbf{r})-\rho_{0}\right] c\left(\mathbf{r}, \mathbf{r}^{\prime} ; \rho_{0}\right)\left[\rho\left(\mathbf{r}^{\prime}\right)-\rho_{0}\right]+F_{\text {uni }}
$$

where $F_{\text {uni }}$ is the free energy of completely uniform liquid. More general functionals also have been constructed and used [19]. Crystallization would be described by a periodic $\rho(\mathbf{r})$, but one can also use such functionals to examine the stability of any conceivable state with persistent aperiodic density. In view of the mostly harmonic nature of individual cages, we can employ the following variational density profile to dress any mechanically sta- 


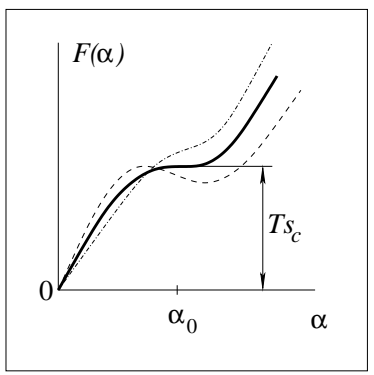

FIG. 7: This is a schematic graph of the free energy density of an aperiodic lattice as a function of the effective Einstein oscillator force constant $\alpha$ ( $\alpha$ is also an inverse square of the localization length used as input in the density functional of the liquid. Specifically, the cureves shown characterize the system near the dynamical transition at $T_{A}$, when a secondary, metastable minimum in $F(\alpha)$ begins to appear as the temperature is lowered.

ble zero temperature structure:

$$
\rho(\mathbf{r}) \equiv \rho\left(\mathbf{r},\left\{\mathbf{r}_{i}\right\}\right)=\sum_{i}\left(\frac{\alpha}{\pi}\right)^{3 / 2} e^{-\alpha\left(\mathbf{r}-\mathbf{r}_{i}\right)^{2}}
$$

Here $\left\{\mathbf{r}_{i}\right\}$ represent the atomic coordinates of a particular, but generic aperiodic lattice characterized by some average density $n \equiv 1 / a^{3}$, where $a$ is the average lattice spacing. The variational parameter $\alpha$ is a convenient measure of localization within such a many-particle cage. While nonzero $\alpha$ characterizes a localized regime, where transient local quasiharmonic environment forms, the same function is also capable of describing the completely delocalized regime of the uniform liquid by taking $\alpha=0$. Singh, Stoessel, and Wolynes [16] established that at sufficiently high density, at a given temperature called $T_{A}$, the free energy from Eq.(3) develops a metastable minimum as a function of $\alpha$, see Fig.7. The transition is very similar to a spinodal crystallization, but to an aperiodic structure. Similar results were obtained earlier by Stoessel and Wolynes [15] using a selfconsistent phonon approach. The density functional and self-consistent phonon approaches both suggest that at $T_{A}$, metastable structures form. This metastability indicates there would be a corresponding barrier demarcating one metastable minimum in the entire set of such minima from the uniform liquid state. The emergence of the minimum appears as a first order transition, where $\alpha$ plays the role of an order parameter, whose value changes discontinuously, during the transition, from $\alpha=0$ to $\alpha=\alpha_{0}$.

The quantity $1 / \sqrt{\alpha_{0}}$ measures the extent of vibrational motion within a local metastable structure. Motions of any greater extent, at the mean-field level, signify a transition between thermodynamically distinct states. The quantity $1 / \sqrt{\alpha_{0}}$ is the vibrational amplitude at the mechanical stability edge, fully analogous to the famous Lindemann length for ordinary crystals [14]. We will denote it as $d_{L}$ :

$$
d_{L}=1 / \sqrt{\alpha_{0}} .
$$

The Lindemann ratio $d_{L} / a$ is roughly one-tenth for all crystals [20-22], and a similar value emerges from the microscopic calculations for aperiodic structures. This value is also consistent with the magnitude of the plateau in the neutron scattering time correlation function [12]. While in terms of $\alpha_{0}$ the transition is first order, there are many aperiodic states randomly distributed in free energy. We therefore call this transition the "Random First Order Transition" (RFOT) because, while first order in $\alpha$, it results in forming many random (infinite lifetime) "phases" which are distinct both morphologically and spatially. Because of the multiplicity of states there is no latent heat but instead at the mean field level, there would be a heat capacity discontinuity much as occurs when a liquid falls out of equilibrium.

Even though the metastable minimum of $F(\alpha)$ is higher in free energy than the uniform liquid state, we realize that the $F(\alpha)$ curve was computed for a single (typical) aperiodic lattice. The full liquid free energy available at $\alpha_{0}$ incorporates the multiplicity of all such (typical) aperiodic states, i.e. $e^{s_{c} N}$ for a region encompassing $N$ molecules. The multiplicity of aperiodic states at the "spinodal" temperature $T_{A}$ obviously must imply the localized minimum is metastable. Whether at a sufficiently low temperature it can reach $F_{\text {uni }}$ and thus imply the existence of an observable thermodynamic state, is not immediately obvious. Yet several rigorous arguments suggest this is indeed the case. Kirkpatrick and Wolynes [23] showed that the mode-coupling viscosity catastrophe, which can be obtained by summing recollision events, also corresponds to the same transition at $T_{A}$ predicted by self-consistent phonon and variational approaches. They noticed the connection with Potts spin glasses. Kirkpatrick and Thirumalai clarified this relation which KW had explicitly showed only in high dimension for fluids $[24,25]$. Like the approximate treatment of the fluid system, the exactly soluble mean-field Potts glass also exhibits a dynamic transition at a temperature $T_{A}$ above its thermodynamic glass transition. Kirkpatrick and Wolynes [26] went on to show that these infinite range Potts spin models, too, exhibited a Kauzmannlike entropy crisis at the lower thermodynamic temperature $T_{K}$. They also suggested that for finite range Potts spin glasses and supercooled liquids the $T_{A}$ transition would be smeared by droplet-like excitations driven by the configurational entropy. These "entropic droplet" excitations would provide the route to equilibration below the mean field dynamical temperature that corresponds to the mode coupling transition. Their argument also explained why the dynamic $T_{0}$ and $T_{K}$ should be the same. These entropic droplet excitations also form the basis of the microscopic developments of RFOT theory that in recent years, have explained supercooled liquid and glasses quantitatively. We will describe entropic droplets in a somewhat different language in the following section. 
The said mean field picture, which employed density functional ideas to treat the fluid aspects and the analogy to the exactly solvable Potts spin glasses, has been developed more formally using the methods of replica field theory. By making reference to the molecular fluid configuration at one time as a fiducial structure, the replica method can be used to construct stability criteria and develop approximations for the configurational entropy directly [27]. Numerous analyses using these tools confirm the earlier mean field developments. These more explicit methods have also been tested near the dynamic transition by computer simulations [28]. The reformulation of the theory using replicas also has allowed the activated events to be studied in a systematic way when the range of the interactions is finite but large [29-31]. These mathematically controlled developments increase the confidence in the simpler constructive arguments for structural glasses that we will describe below. Another direction in which the microscopic calculations can be taken is to explore the molecular origins of the configurational entropy. Hall and Wolynes recently used these approaches to explain why network materials with more constraints (like $\mathrm{SiO}_{2}$ ) behave like "strong" liquids while simple van der Waals systems without bonding constraints are more fragile [32].

It is important to note that within the RFOT theory, detailed microscopic calculations starting from the intermolecular forces can in fact be carried out. Presently, only the mode coupling theory can make a comparable claim. Mode coupling theory (MCT) is not an orthogonal approach, however. As Kirkpatrick and Wolynes pointed out in 1987 [23] the two approaches are effectively equivalent near the dynamical crossover. Recent successes of $\mathrm{MCT}$ in describing re-entrant glass transitions in attractive colloids, etc. thus buttress the general RFOT picture from a microscopic viewpoint. Microscopic calculations set the stage for the RFOT theory. Much as in thinking about conventional phase transistion, it is important, however, to distinguish the main RFOT ideas from the liquid state engineering details for specific systems. It is clear, for example, that the deep supercooled regime of van der Waals liquids probes intermolecular forces at a more intimate length scale than near the critical point. Time-honored prescriptions based on perfectly hard potentials thus will break down. This breakdown is most clearly evidenced by the experimental fact that the isochoric activation energy of the viscosity is not zero below $T_{A}$, while it is nearly so above that point. Just as for studies of crystals [33], the popular Weeks-ChandlerAndersen prescription [34], for dividing the potential, requires re-examination in deeply supercooled liquids.

\section{B. The Library of Local States}

In mean field theory, the lifetime of an aperiodic structure is infinite. But in reality the lifetime of a system with finite range interactions is finite because rearrange- ments can occur independently within regions of finite spatial extent. The number of relaxation events per unit time scales with the system size. A mole of water will undergo roughly $10^{39}$ barrier crossing events a second. These events are local in character in that they affect each other little beyond some critical distance.

The locality of structural processes in supercooled liquids is intrinsic in the entropic droplet concept. Recently their quantitative aspects have been constructively established within the RFOT theory [35, 36]. Our present discussion will be based on the "library" construction of local configurations [37], which makes the ideas more transparent than in earlier discussions and also allows the treatment of the "aging" regime. This library construction is essential for the latter far-from-equilibrium case. An elegant formal analysis along similar lines has been made by Bouchaud and Biroli [31]. This construction can also be quantized to deal with the phenomena of cryogenic temperatures [38]. In the library construction, one first averages over the vibrational modes of the supercooled liquid. These equilibrate generically in a time less than a picosecond. The variational ansatz used in density functional theory from Eq.(3) operationally defines such an averaging. The lifetimes of the resulting aperiodic crystal states depend on the size of the region. For the library construction to be strictly valid, the processes must be slower than the sound modes of similar length by three orders of magnitude or so. Fortunately, this criterion covers most of the dynamical range accessed by supercooled melts, except perhaps for the high frequency processes referred to as the Boson peak in liquids [39]. Transitions between the aperiodic crystal states defined above give rise to the configurational entropy.

To convert from one state to another state we must consider not only direct paths connecting one structure to a neighboring one; but most importantly, one must examine all thermally realizable dynamically connected sequences containing many structural states. By "dynamically connected" we mean the trajectory is a sequence of processes with small barriers that involves a translation of a single bead by a distance not exceeding the Lindemann length $d_{L}$. The lowest energy path leading to a thermally representative liquid state will define the most probably escape trajectory from the initial state. The highest free energy point along the path will determine the bottleneck, or the critical value of the progress coordinate. The energy at the bottleneck will determine the barrier height and will thus give the activation part of the escape rate. A very nice example of a computational study of such a sequence is provided by Saksaengwijit and Heuer [40]. They show that even in high temperature silica, whose rates are nearly Arrhenius, reconfiguration events proceed through several steps, see Fig.9.

Now, the totality of the aperiodic states that could be constructed with a region of fixed boundaries forms a "library" of local states. This construction is illustrated in Fig.8. In this figure, the circles do not signify the instantaneous positions of the molecules but rather their most 


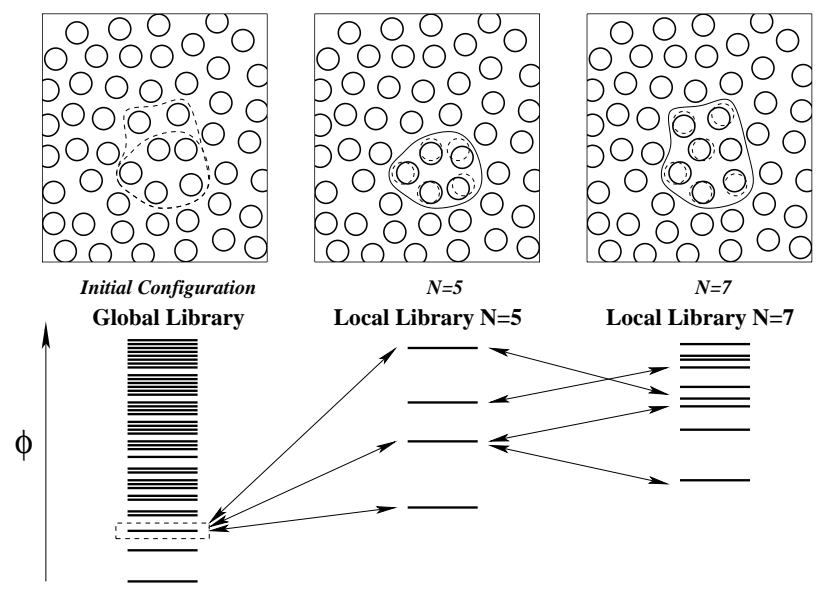

FIG. 8: This figure is taken from [37]. In the upper panel on the left a global configuration is shown, chosen out of a global energy landscape. A region of $N=5$ particles in this configuration is rearranged in the center illustration; this subset is taken from another aperiodic state or from a different location of the same liquid state. The original particle positions are indicated with dashed lines. A larger rearranged region involving $N=7$ particles is connected dynamically to these states and is shown on the right. In the lower panel, the left most figure shows the huge density of states that is possible initially. The density of states found in the local library originating from a given initial state with 5 particles being allowed to move locally is shown in the second diagram. These energies are generally higher than the original state owing to the mismatch between the two structures. The larger density of states where 7 particles are allowed to move is shown in the right most part of this panel. As the library grows in size, the states as a whole are still found at higher energies but the width of the distribution grows. Eventually with growing $N$, a state within thermal reach of the initial state will be found. At this value of $N^{*}$ we expect a region to be able to equilibrate.

probably positions after vibrational averaging. Further, the circles correspond to effective individual structural units, or "beads". Chemical intuition often makes clear how to decompose conceptually a molecule into beads. A rigid chemical group, a single benzene ring, or a compact side-chain in a polymer will usually constitute an independently moving unit. The volumetric bead density can be quantified unambiguously by comparing with the fusion entropy, if the corresponding crystal exists, since bead motions are also frozen in the crystal; see the detailed discussion in [41]. At any rate, a bead is usually a few angstroms in size.

Consider an initial configuration spanning some extended region. Its "intrinsic" bulk energy can be written:

$$
\Phi_{\text {in }}^{\text {bulk }}=E_{\text {in }}-T S_{\text {vibr }},
$$

which combines the energy proper of the particular arrangement of the most probable molecular coordinates with the free energy of the high frequency vibrations.

This state may move to some other thermally representative aperiodic crystal state over the same region,

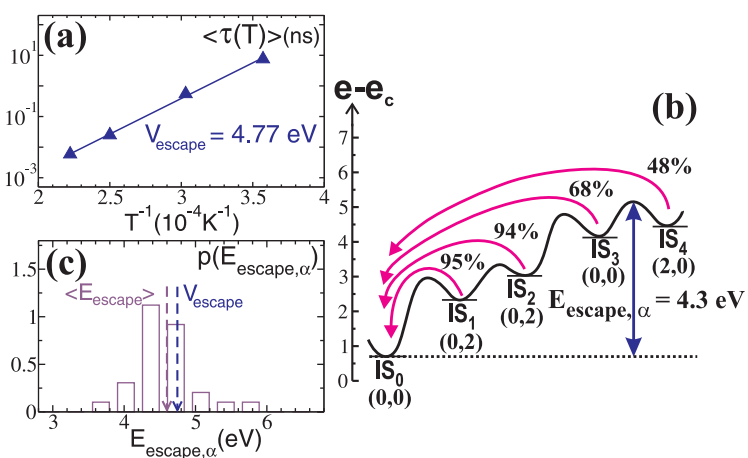

FIG. 9: Even in "strong" liqiuds, reconfigurations occur through a series of steps as in the library constuction. Saksaengwijit and Heuer have delineated these events in silica. Their results are shown with their Fig.4 [40]. Panel (a) shows the temperature dependence of the average waiting time in the low energy inherent structures of simulated silica. In (b), a specific escape trajectory from an inherent structure is represented. The percentages indicate how often such a structure recurs to its initial state. Panel (c) gives the distribution of escape barriers from IS.

labelled by a dummy index $j$ whose bulk energy is:

$$
\Phi_{j}^{\mathrm{bulk}}=E_{j}-T S_{\mathrm{vibr}}
$$

Choose a boundary $\mathcal{B}$ encompassing $N$ beads somewhere within the initial structure, and remove its contents. We then cut an identically shaped region out of the structure $j$ and paste this region in the void in the initial structure. Call the modified region of the initial state a "droplet". The free energy of this trial configuration is:

$$
\phi_{j}-\phi_{i n}=\Phi_{j}^{\mathrm{bulk}}-\Phi_{i n}^{\mathrm{bulk}}+\Gamma_{j, i n}
$$

This free energy includes a mismatch penalty $\Gamma_{j, i n}$ because no matter how carefully we placed the surface $\mathcal{B}$ in the structure $j$, the contents of the latter region were vibrationally averaged with a constrained surrounding different from the one in the initial state and thus some "elastic energy" must be paid. The alternative state inside the droplet may be thought of as the original molecules somewhat displaced from their original positions. The cut-and-paste routine realizes a finite number of thermodynamically distinct droplet states. This finite number is given by the configurational entropy, and scales exponentially with the droplet size: $e^{s_{c} N}$. In the central panel of Fig.8, we show, for illustration, just the subset of droplet states available at size $N=5$. This subset would constitute an astronomically minute fraction of the whole set of states available to an extended sample. Now, consider the droplet states at different, but close sizes. Some states at both sizes will be structurally very close, implying high degree of dynamical connectivity. Sequences of such dynamically connected droplet states constitute escape trajectories from a given initial 
extended state to some other extended aperiodic crystal state. Because one may associate with any of those sequences of droplet states a sequence of droplet sizes, one may therefore speak of nucleation of one aperiodic crystal state within another and of the corresponding propagation of a domain wall separating those two aperiodic crystal states. In this way, the mismatch penalty may be thought of as the domain wall tension.

Because of the surface penalty, the lowest energy, per particle, in a droplet library is likely to be well above the energy, per particle, in the initial extended state. Still, the energies within such finite subsets are always distributed, roughly in a Gaussian fashion. The lowest energy in the subset, it turns out, is determined by a competition between two factors: On the one hand, with the growing droplet size, the mismatch penalty will increase thus shifting the bulk of the distribution of the subset energies upwards. On the other hand, the weight of the distribution, i.e. $e^{s_{c} N}$, increases exponentially with $N$, so that the lowest energy state will be found at an increasingly lower position. Eventually, at large enough droplet size $N^{*}$, the latter trend will compensate the former, so that the droplet will be guaranteed to have the energy per particle equal to that of an arbitrarily extended state. Thus to a region of size $N^{*}$ or larger, all thermodynamically relevant bulk liquid states will be available; i.e. such a region is expected to be typical of equilibrium at the temperature $T$. A supercooled liquid is a mosaic of aperiodic crystals! In the following we will put this qualitative discussion in formal, quantitative terms.

Mismatch Penalty between Aperiodic Crystal States. To a first approximation from the density functional viewpoint, computing the mismatch energy is simply a matter of counting up the missing interactions from some unsatisfied local contacts in an interface region. The mismatch penalty would thus scale with the interface area itself, times the energy of the unsatisfied bond: $\Gamma \propto \epsilon r^{d-1} l_{\text {micro }}$, where $\epsilon$ and $r$ are the interface energy density and the region size respectively [26]. This is also the result of instanton calculations based on replica methods $[42,43]$. However, the situation is a bit more complicated as we must recognize that some of the states in the library will in fact match much better than do others because they are partially random. The scaling of thermal averaged $\Gamma$ with $N$ will thus be weaker than expected for interfaces between entirely distinct phases. The fraction of the better matching configurations is significant enough to actually partially short-circuit the conventional surface tension, renormalizing it to a smaller value. From an elastic theory point of view, we expect the displacement fields due to the local structural rearrangements in our aperiodic structures not to exhibit long range correlations. Accordingly, the deviation of total strain within a region of size $M$ from its average value would scale with the usual $\sqrt{M}$, and is of either sign with equal probability. This means one may generically impose a random external field with the usual Gaussian statistics to lower the energy of a region of size $M$ by an amount scaling with $\sqrt{M}$. A curved interface between two states will distort so as to lower the local free energy to take advantage of this random contribution. The energy compensation will scale, again, as the square root of the variation of the volume occupied by either of the two phases, due to the boundary distortion. The final shape of the interface will be determined by a competition between this stochastic energy compensation and the cost of increasing the area of a flat interface. Consistent with this, the scaling of surface tension with $r$ differs from the conventional $r^{(d-1) / d}$. This situation is analogous to the problem of the interface between the spin-up and spindown regions in the Random Field Ising Model (RFIM), which has been treated by Villain [44]. This analogy was explicitly exploited by Kirkpatrick, Thirumalai, and Wolynes (KTW) [35] in deducing how the droplet interface tension scales with the droplet size. (One may in fact show that the two problems can be mapped onto each other, with the error not exceeding the discrepancy between the microcanonical and canonical averages [45].) A pleasant dividend of this argument is that the hyperscaling relation connecting the heat capacity and the mosaic length scaling near $T_{K}:(2-\alpha=\nu d)$, - is restored [35]. The KTW argument suggests the effective surface tension coefficient is renormalized according to:

$$
\sigma(r) \sim \sigma_{0}(r / a)^{-(d-2) / 2},
$$

where $\sigma_{0}$ is the surface tension coefficient at the molecular length scale. In all spatial dimensions $d>2$, the scaling of the resulting mismatch free energy

$$
\Gamma \propto r^{d / 2}
$$

is numerically inferior to the conventional $r^{(d-1) / d}$ scaling, but of course this is quantitative only for sufficiently large interfaces. It also follows that the thickness of the distorted interface scales with the radius itself $[35,44]$ consistent with our à priori expectation that the mismatch energy is determined by the subsets of static structures that smoothly interpolate between two generic extended aperiodic states. It may be said that an interface between two thermally averaged aperiodic crystals is, strictly speaking, not thin but is always "wetted" by other states.

On short length scales, the coefficient $\sigma_{0}$ in Eq.(9) must correspond to the mismatch penalty at the molecular length scale. This suggests a way in which $\sigma_{0}$ can be estimated in zeroth order, at the molecular length-scale. The mismatch requires the particle at the interface to still be within the Einstein oscillator localization volume $d_{L}^{3}$, instead of its volumetrically available space $a^{3}$. Yet in the free energy functional it does not receive the full benefit of its neighbors' being localized (only half of them are) and thus staying out of the way. Since these free energies must balance at $T_{K}$, there is an additional mismatch penalty on this scale that is related to the localization entropy $\sigma_{0} \sim \frac{1}{2} k_{B} T \ln \left(a^{3} / d_{L}^{3}\right)=(3 / 4) k_{B} T \ln \left(a^{2} / d_{L}^{2}\right)$. This expression for $\sigma_{0}$ contains all the essential parameter scal- 
ing, but obviously there may be some numerical uncertainty in applying the long distance scaling of $\sigma(r)$ all the way down to the shortest lengths. Nevertheless, by virtue of the slow, logarithmic dependence on the constants, along with the near universal value of the Lindemann ratio $d_{l} / a=0.1$, this argument suggests $\sigma_{0}$ is a universal multiple of the glass transition temperature itself. This is the key to the later quantitative results obtained in the RFOT theory. Using the density functional approximation of Eqs.(3) and (4), Xia and Wolynes specifically obtained the surface tension at the molecular scale [36]:

$$
\sigma_{0}=\frac{3}{4} \frac{k_{B} T}{a^{2}} \ln \left[\left(a / d_{L}\right)^{2} / \pi e\right], \text { in } \sigma(r)=\sigma_{0}(a / r)^{1 / 2} .
$$

This value works very well quantitatively for real materials. The chemical universality of $\sigma_{0}$ reminds one of Turnbull's rule used in treating crystallization that empirically states the tension between a periodic crystal and its melt is a universal multiple of $T_{m}$ [46]. It is convenient to write the mismatch free energy in terms of the number of reconfigured particles: $N \equiv(4 \pi / 3)(r / a)^{3}$ :

$$
\Gamma=\gamma \sqrt{N}, \text { where } \gamma \equiv \frac{2 \sqrt{3 \pi}}{2} k_{B} T \ln \left[\frac{\left(a / d_{L}\right)^{2}}{\pi e}\right] .
$$

While the mismatch energy $\Gamma_{i n, j}$ is doubtless distributed, as long as we are dealing with typical liquid states, we can use its typical value $\Gamma$.

\section{Activated Motions between Local States}

We see, via the library construction, that if too small a region is reconfigured, even the lower energy paths will have a monotonically increasing energy with the size. But as the region is made larger, some fraction of paths will curve down in energy. At sufficiently large size, there will always be a trajectory ending at an energy within the thermally relevant liquid energy range. In computing the typical escape rates from a liquid state, one therefore only needs to escape by passing through droplet configurations with the size corresponding to the lowest flux. Formally averaging the escape flux over the ensemble of transition state droplets yields [37]:

$$
\begin{aligned}
k & =\tau_{0}^{-1} \int\left(d \phi_{j} / c_{\phi}\right) e^{S_{c}\left(\Phi_{j}^{\text {bulk }}\right) / k_{B}} e^{-\left(\phi_{j}-\phi_{i n}\right) / k_{B} T} \\
& \simeq \tau_{0}^{-1} e^{S_{c}\left(\Phi_{o p t}^{\text {bulk }}\right) / k_{B}} e^{-\left(\phi_{o p t}-\phi_{i n}^{l i b}\right) / k_{B} T} \\
& \equiv \tau_{0}^{-1} e^{S_{c}\left(\Phi_{e q}^{\text {bulk }}\right) / k_{B}} e^{-\left(\phi_{e q}-\phi_{i n}^{l i b}\right) / k_{B} T} .
\end{aligned}
$$

The intrinsic activation free energy represents a critical droplet-configuration energy from Eq.(8) and the factor $e^{S_{c}\left(\Phi_{j}^{\text {bulk }}\right) / k_{B}}$ gives the multiplicity of liquid configurations at the energy $\phi_{j}$. ( $c_{\phi}$ is a normalization constant.) The energy $\phi_{\text {opt }}$, maximizing the integral, must be assigned the equilibrium energy value. Consequently, the $\log$ of the multiplicity, $S_{c}\left(\Phi_{e q}^{\text {bulk }}\right)$, is nothing but the configurational entropy at equilibrium, which can be measured by calorimetry: $S_{c}\left(\Phi_{e q}^{\text {bulk }}\right)=S_{c}$.

The $N$ dependence of all the parameters in Eq.(13) becomess obvious from Eq.(8) upon recalling that $S_{c}$ and $\Phi^{\text {bulk }}$ are bulk parameters and scale with $N$ itself: $S_{c}=N s_{c}, \Phi^{\text {bulk }}=N \phi^{\text {bulk }}$. One thus obtains a simple expression for the typical free energy profile during a structural rearrangement after optimizing with respect to the saddle point energy. It varies with size as:

$$
F(N)=\left[\phi_{e q}^{\text {bulk }}(T)-\phi_{i n}^{\text {bulk }}(T)\right] N+\gamma \sqrt{N}-T s_{c} N .
$$

Here, we have used Eq.(8). $F$ does reach down to arbitrarily low energy states, but to escape, one needs to pass first over the maximum, which gives the typical barrier $F^{\ddagger}$ for reconfiguration from a given initial state. When $F=0$, a typical state has already been reached.

Consider supercooled liquids equilibrated above the glass transition temperature $T_{g}$. The initial liquid state is thermodynamically typical of the temperature $T$ thus $\phi_{i n}^{\text {bulk }}(T)=\phi_{e q}^{\text {bulk }}(T)$. The resulting nucleation profile is quite simple:

$$
\left.F(N)\right|_{T>T_{g}}=\gamma \sqrt{N}-T s_{c} N .
$$

Clearly, structural transitions are driven by configurational entropy alone! Eq.(15) immediately gives an inverse scaling of the most probable relaxation barrier with the configurational entropy density that automatically yields the Vogel-Fulcher law:

$$
F^{\ddagger}=\frac{\gamma^{2}}{4 s_{c} T}=\frac{\gamma^{2}}{4 \Delta c_{p}\left(T-T_{K}\right)},
$$

Here we used the specific form (2) for the configurational entropy. Note that if it were not for the surface tension renormalization, see Eq.(10), the $s_{c}$ dependence in the denominator of the middle expression would be quadratic, not linear. The inverse scaling of the relaxation barrier with the configurational entropy was derived by KTW [35], but was proposed originally by Adam and Gibbs (AG) [47], who stipulated that there be a smallest rearranging unit in a liquid. This AG unit would be characterized by two configurations, whose size was assumed independent of temperature. The Adam-Gibbs argument does not reconcile how the existence of such a special fixed size is compatible with that argument's use of a temperature dependent, extensive configurational entropy. In contrast, within RFOT theory the length scale of activation is determined by the underlying Hamiltonian and varies with temperature. The RFOT thoery precisely predicts the way in which the critical size of the nucleation barrier scales in Ref. [35]:

$$
r^{\ddagger} \propto\left(N^{\ddagger}\right)^{1 / 3} \propto \frac{1}{s_{c}^{2 / 3}} \propto \frac{1}{\left(T-T_{K}\right)^{2 / 3}} .
$$

This scaling law is consistent with a specific heat discontinuity and the usual hyperscaling relation for continuous transitions [35]. RFOT theory directly shows the kinetic and thermodynamic anomalies in supercooled melts 
are intrinsically related. Furthermore, given the value of $\sigma_{0}$ from Eq.(11), Xia and Wolynes established an amazingly simple relation between the kinetic fragility and the heat capacity discontinuity:

$$
D=32 . / \Delta c_{p}
$$

The universal numerical constant 32 . comes from the numerical constants in the argument and the universal value of the Lindemann ratio. $\Delta c_{p}$ is the heat capacity jump per bead. Owing to the universality of the Lindemann ratio, RFOT theory implies the material dependence of the fragility comes essentially from the heat capacity only. Since the Lindemann length enters under the logarithm, its small variations do not affect the constant significantly. In any event, a recent argument by Lubchenko [48] shows the Lindemann ratio $d_{L} / a$ would be expected to vary at most my $10 \%$ in supercooled liquids.

The present argument assumes the liquid to be sufficiently deeply supercooled so that deviations from the activated picture are small. This is true already when relaxations are about three orders of magnitude slower than the times of vibrational temperature equilibrium. The activated picture thus applies at $\tau>10^{-9}$ sec, which covers most of the liquid dynamic range. At higher temperatures, collisional effects on the viscosity become important. The agreement of the relation of Eq.(18) between $D$ and $\Delta c_{p}$ with available data is impressive. Nevertheless, to avoid fitting ambiguities, it appears best to use the measured fragility index near $T_{g}$ : $m=T\left[d \log _{10} \tau(T) / d(1 / T)\right]$. The index $m$ scales roughly inversely proportionally with $D$, but is less dependent on the fitting ambiguities arising from the crossover to the high temperature region. Like $D, m$ also follows from $\Delta c_{p}$ without adjustable parameters. Fig.6 shows how theory and experiment compare for the predicted $m$ from thermodynamics and the measured kinetic values. There are a small number of outliers. In examining those exceptions one must bear in mind, however, the RFOT theory applies strictly only to purely amorphous and fully equilibrated melts. Deviations from the RFOT predictions are expected when samples exhibit partial crystallinity, often present in polymers, or other types of local order, as in decalin or in alkali borates (see e.g. [49]),

Whether such local order effects exist may actually be judged, to some extent, using another simple result that follows from the RFOT theory: Near $T_{g}$, relaxations are strictly activated, implying the typical relaxation barrier relative to the temperature depends only logarithmically on the relaxation rate: $\left(F^{\ddagger} / T\right)_{T_{g}}=\ln \left(\tau / \tau_{0}\right)$. The quenching rate in the laboratory is limited by the sample's heat conductance, on the faster side, and the experimenter's patience and stable temperature conditions, on the longer side. Combining a one hour time scale with Eqs.(12) and (16), RFOT predicts the configurational entropy at $T_{g}$, per bead, should be [36]:

$$
s_{c}\left(T_{g}\right) \simeq 0.8,
$$

depending only logarithmically on the ratio $\tau / \tau_{0}$. The

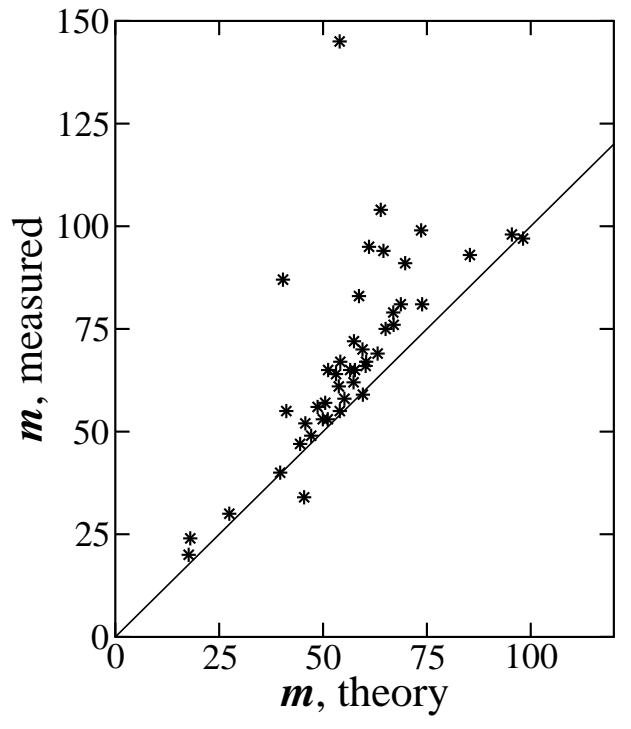

FIG. 10: The horizontal axis shows the value of fragility as computed from the thermodynamics by the RFOT theory, and the vertical axis contains the fragility directly measured in kinetics experiments. Here $m$ is the so called fragility index, defined according to $m=T\left[d \log _{10} \tau(T) / d(1 / T)\right] . m$ is somewhat more useful than the fragility $D$, because deviations from the strict Vogel-Fulcher law, $\tau=\tau_{0} e^{D T_{K} /\left(T-T_{K}\right)}$, are often observed, see text. $m$ essentially gives the apparent activation energy of relaxations at $T_{g}$, in units of $T_{g}$, it is roughly an inverse of $D$. In evaluating $m$ theoretically, one needs to know the size of the moving unit, or "bead", in each particular liquid. The latter can be estimated using the entropy loss at crystallization and scaling it to its Lennard-Jones value [41], resulting in $m_{\text {theor }} \propto \frac{\Delta c_{p}\left(T_{g}\right) T_{g}}{\Delta H_{m} s_{c}^{2}\left(T_{g}\right)} \propto \frac{\Delta c_{p}\left(T_{g}\right) T_{g}}{\Delta H_{m}}$, in view of the near universality of $s_{c}\left(T_{g}\right)$ (see text). This figure is taken from [50].

latter time ratio, at $T_{g}$, is very large: $10^{16}$ or so, rendering the estimate above nearly universal. A brief look at the configurational entropies of polymers, such as in Fig.4 of Roland at el. [51] immediately gives away there are many partially crystalline polymers. For example, PVC has $s_{c}\left(T_{g}\right) \simeq .1$ clearly indicating a large degree of crystallinity, consistent with X-ray determinations of the latter.

Another important universality predicted by the RFOT theory is the size of the cooperatively rearranging region at the glass transition temperature. For the droplet size $N>N^{*}$, where $F\left(N^{*}\right)=0$, one expects all thermally relevant liquid states have become available; there is no further "growth" of an alternate phase. Typically then $N^{*}$ beads are reconfigured during a typical relaxation event. Using

$$
N^{*} \equiv(\xi / a)^{3},
$$

one easily finds [36]:

$$
\left.\xi\right|_{T=T_{g}}=5.8 a,
$$


for $\tau / \tau_{0}=10^{17}$, i.e. at the relaxation time of the order an hour. This cooperativity length scale $\xi$ is not structural, but dynamic, and hence must be probed by nonlinear dynamic experiments. Because any region of size $\xi$, but no smaller, may relax, we can regard a supercooled liquid as a "mosaic" [36] of cooperatively rearranging regions or "entropic droplets". Like the critical radius $r^{\ddagger}$, the dynamical heterogeneity of length scale $\xi$ scales according to

$$
\xi \propto \frac{1}{\left(T-T_{K}\right)^{2 / 3}} .
$$

Eqs.(21) and (22) are arguably the most specific microscopic predictions of the RFOT theory; they are certainly consistent with the earlier semi-quantitative results of the 4D exchange NMR experiments by Tracht at el. in PVAc [52]. Concurrently with the publication of the theory or a bit later, other types of non-linear studies have been performed on several supercooled liquids which all quantitatively confirm the RFOT prediction. These studies include experiments using nanometer dielectric probes by Russell and Israeloff [53]. Deviations of the hydrodynamics of small probes from the Stokes-Einstein relation [54] are also consistent with this length scale as earlier predicted by Xia and Wolynes [55], who showed deviations from the Stokes-Einstein relation are expected for probes smaller the length $\xi$. These deviations are especially notable in that they explicitly show the cooperativity length is temperature dependent [55]. Very recently, evidence of a dynamic cooperative length, which increases with lowering the temperature, have been obtained using a rigorous inequality based on the non-linear susceptibility. Berthier at el. [56], again, decisively confirm the result in Eq.(21). Finally, while the detailed temperature dependence of the cooperativity length is somewhat modified by the mentioned barrier softening effects, the universality from Eq.(21) is robust, in the whole range of liquid fragilities [41].

Eq.(15) only gives the typical droplet nucleation profile. Specific activation paths will reflect local variations of the liquid landscape, i.e. they depend on the initial local configuration. These variations are encoded in the local density of states (DOS) that can be connected to the original configuration. A higher than average density of states implies more configurations are available, leading to a lower activation free energy barrier and a smaller number of molecules participating in a structural transition, see Eqs.(16) and (17). Local variations in the DOS correspond to fluctuations of the configurational entropy. Entropy fluctuations, on the other hand, follow from the standard formula $\left\langle\left(\Delta S_{c}\right)^{2}\right\rangle=\Delta C_{P}$. Only the configurational part of the heat capacity, as given by the heat capacity jump at the glass transition, enters. Just this logic was followed in Ref.[57]. At this level of approximation, the entropy fluctuations, which are approximately gaussian (surely, for $N^{*}>6$ ), lead to a nearly gaussian distribution of barriers, via Eq.(16). The ratio of the barrier distribution width to its most probable value de-

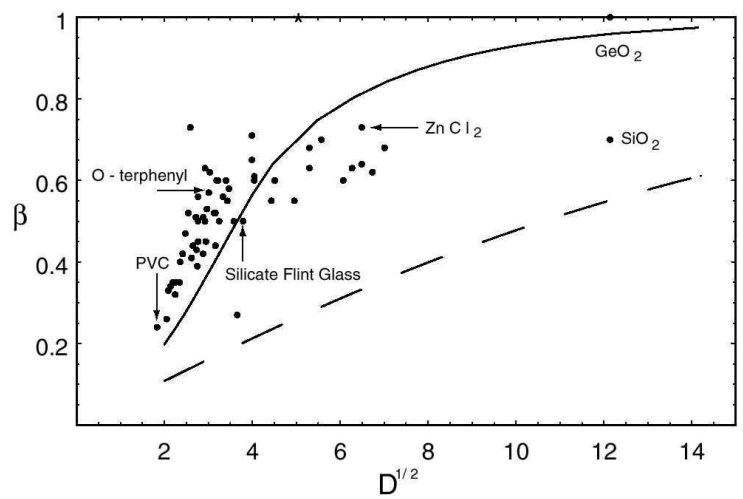

FIG. 11: This figure shows the correlation between the liquid's fragility and the exponent $\beta$ of the stretched exponential relaxations, as predicted by the RFOT theory, superimposed on the measured values in many liquids taken from the compilation in Ref. [58]. The dashed line is based on a simple gaussian barrier distribution, with the width mentioned in the text. The solid line takes into account the averaging effect of environmental rearrangments surrounding a mosaic cell, so that the barrier distribution to the right of the most probable value is replaced by a narrow peak of the same area; the peak is located at that most probable value. This figure is taken from [57].

pends only on the liquid's fragility [57]:

$$
\frac{\delta F^{\ddagger}}{F_{\mathrm{mp}}^{\ddagger}} \simeq \frac{1}{2 \sqrt{D}} .
$$

As a result of the barrier distribution, bulk measurement of relaxations will produce non-exponential time decay, or, non-Lorentzian profiles in the frequency domain. The former is often fitted with a stretched exponential:

$$
p(t)=e^{-\left(t / t_{0}\right)^{\beta}} .
$$

Assuming the barrier distribution is purely gaussian, one indeed recovers a decay profile closely resembling the stretched exponential form, where the corresponding exponent $\beta$ is related to the fragility in the following simple manner [57]:

$$
\beta \simeq\left[1+\left(\delta F^{\ddagger} / k_{B} T\right)^{2}\right]^{-1 / 2} .
$$

This correlation is shown by the dashed curve in Fig.11. While already qualitatively consistent with experimental data, further improvement in agreement is immediately achieved upon realizing that if a particular region happens to be relatively short on alternative structural states, a slightly distinct, and hence overlapping (!), region is not likely to be so disabled. Since the environment of the original "slow" domain will change such a slow region will relax sooner than expected. Xia and Wolynes suggested therefore the barrier distribution, for $\alpha$-relaxation, should be asymmetric, with relatively less weight on the high barrier flank. The simplest way 
to incorporate the above considerations into the theory is to use the original gaussian distribution, but with the slow half of that distribution replaced by a delta function carrying the same total weight, and centered at the most probable value of the original gaussian. This approximation introduces no new parameters. Using this simple form still leads to a correlation of $\beta$ with $D$ and reproduces the empirically known correlation between $\beta$ and $D$ quantitatively [57], see the solid line in Fig.11. The RFOT theory clearly predicts the degree of nonexponentiality is temperature dependent, consistent with findings of Dixon and Nagel [11].

\section{Dynamics near the Crossover}

The temperature $T_{A}$ is a mean-field spinodal where one expects a siginificant barrier softening, as $T_{A}$ is approached from below. Above $T_{A}$ transport is no longer strictly activated. Instead, weakly correlated collisions become the dominant contributor to the liquid's viscous response. The barrier softening effects have been quantitatively assessed by us [41]. These effects are particularly important at viscosities below 10 Poise or so, for all considered substances.

For salol, the theoretically derived contribution to the relaxation stemming from activated processes is shown as the solid line in the left pane of Fig.12. The theory uses experimentally derived values of the configurational entropy and the high temperature limit of the relaxation times using the prefactor given by collision theory. The softening correction computed by Lubchenko and Wolynes (LW) relies on the idea that at small droplet radii, there is less interface wetting. The correction terms in their analysis involve only one fitting parameter, which is the spinodal dynamical crossover temperature $T_{A}$, where the mean-field barrier between liquid states would vanish. Clearly, at viscosities less than 10 Poise or so, where the theoretical and the experimental curve bifurcate, the transport becomes largely collisional. For several systems, the temperature where the experiment and theory diverge, turns out to lie rather close to the temperature $T_{c}$, where Stickel at el. [59] have found a "kink" in the temperature dependences of relaxation times. Often, two distinct VF forms have been used to fit the data in the two temperature ranges, separated by $T_{c}[59]$.

While the LW analysis takes the temperature $T_{A}$ from fits, a recent argument of Stevenson, Schmalian, and Wolynes [60] provides a microscopic description of the onset of activation-less reconfigurational motions. The overall high free energy profile, Eqs.(14) and (15), comes from the relative scarcity of phase space trajectories that are sequences of low cost local moves. The overall free energy cost should not only include competition between the mismatch energy of mobilizing particles against immovable neighbors and the entropy gain so achieved, but also the number of different ways a connected pattern
Salol

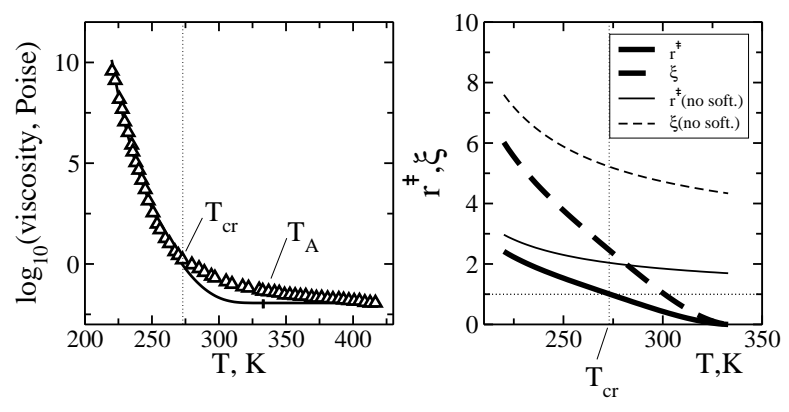

FIG. 12: Experimental data (symbols) for salol's viscosity [59], superimposed on the results of the fitting procedure (line) from [41] are shown. $T_{A}$ is the temperature at which the meanfield barrier vanishes, indicated by a tickmark. The configurational entropy, used in the fitting procedure, was extracted from experiment, see Ref.[8] and Eq.(2). The temperature $T_{\mathrm{cr}}$ signifies a cross-over from activated to collisional viscosity, dominant at the lower and higher temperatures respectively. The temperature is varied between the boiling point and the glass transition. The r.h.s. pane depicts the temperature dependence of the length scales of cooperative motions in the liquid. The thick solid and dashed lines are $r^{\ddagger}$ and $\xi$ respectively. This figure is taken from [41].

of mobile particles can be placed on an aperiodic lattice. Keeping track of such patterns can be done, for instance, by counting contiguous percolation clusters [61] or by counting impenetratable strings emanating from a common origin. Here, again, the probability to find a contiguous cluster grows with increasing configurational entropy. The estimates based on percolation clusters or strings give distinct but comparable values of the critical configurational entropy at which non-activated reconfiguring short-circuits become possible [60]: $s_{c}^{\text {perc }}=1.28 k_{B}$ and $s_{c}^{\text {string }}=1.13 k_{B}$. The corresponding temperature at which a crossover to non-activated transport occurs, is given by

$$
\frac{T_{c}^{\text {perc }}}{T_{K}}=\left(1-\frac{s_{c}^{\text {perc }}}{\Delta c_{p}} \frac{T_{K}}{T_{g}}\right)^{-1},
$$

A similar result is obtained for the "string" transition. Here, the functional form for the configurational entropy from Eq.(2) has been used. In Fig.13, we show the theoretically derived values of $T_{c}^{\text {perc }}$ and $T_{c}^{\text {string }}$, in comparison with the Stickel's $T_{c}$. Note that no adjustable parameters are used in Fig. 13. It is interesting that the mathematics of the string calculation is isomorphic to the Hagedorn transition in the string theories of particle physics [62].

We see that RFOT theory predicts deviations from the strict Vogel-Fulcher dependence of the relaxation times on the temperature. These deviations are expected to occur also at the faster side of the full dynamical range of liquid relaxations. The deviations span about three orders of magnitude, compared with the overall range 


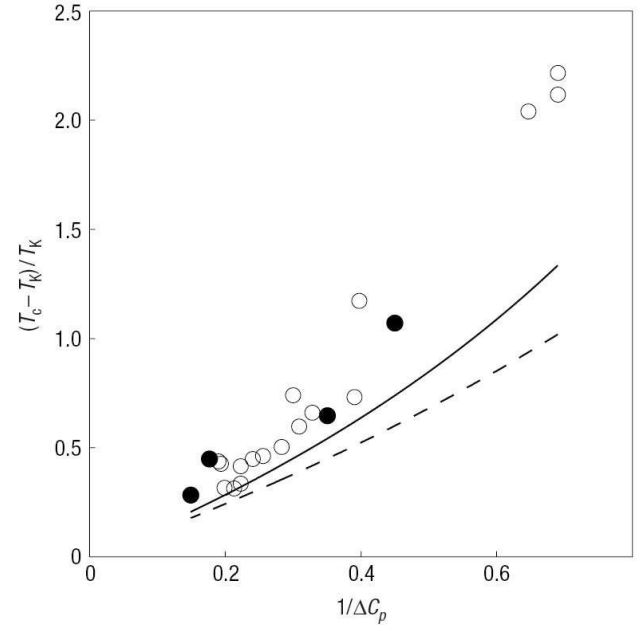

FIG. 13: From Ref.[60]: Predictions for $\left(T_{c}-T_{K}\right) / T_{K}$ based on string-like rearranging regions is a dashed line, and $\left(T_{c}-\right.$ $\left.T_{K}\right) / T_{K}$ based on percolation clusters is the solid line. The experimentally derived crossover temperatures, $\left(T_{c}^{e x p}-T_{K}\right) / T_{K}$, from those materials collected by Novikov and Sokolov [63], are shown as circles with the dark circles referring to polymers.

of $10^{17}$ or so, accessible in experiment. At these faster times, according to Ref.[60], reconfigurations involving non-compact regions become important. This is consistent with "string-like" excitations that had been observed in numerical simulations of liquid dynamics by Glotzer and coworkers [64].

Generally simulations are carried out in the dynamic range where these softening effects are important. Yet, as in the last example, the RFOT theory is indeed consistent with current simulational studies. To be specific, current computational technologies are limited to a dynamic range of nine decades or so. The time step in simulation is roughly a femtosecond, significantly less than a typical collision time. In view of the large system size necessary to avoid boundary effects, at best one produces currently a microsecond run, implying only thermally representative relaxations on a small fraction of a microsecond time-scale can be ascertained. (Equilibrating a liquid in a realistic simulation, in the deeply supercooled regime, must of course be subject to the very same high barriers present in the laboratory melts.) According to a recent discussion of Lubchenko [65], such short times imply the size of the critical nucleus (or the participation number at the saddle point, if you will) is only about $(4 \pi / 3) 1^{3} \simeq 5$ "beads", corresponding to $r^{\ddagger} / a \simeq 1$. In silica, the bead is a fraction of $\mathrm{a} \mathrm{SiO}_{4}$ tetrahedron $[41,66]$, implying a critical region size of 15 atoms or so. This is consistent with the participation ratios, at the barrier top, reported recently by Reinisch and Heuer in simulations characteristic of these short times [67]. According to this discussion, one must be careful so as not to mistake a possible but unrepresentative high barrier trajectory, that could be always found given a high enough temperature, for a thermally representative trajectory that actually describes reconfiguration on the laboratory time scale.

\section{E. Aging}

A liquid is in equilibrium. Ultimately there is no memory of its initially prepared configuration. But when the liquid is cooled faster than it can equilibrate, the system will find itself in a subset of all the states available before the quench began. Which subset depends on the thermal history. The resultant quenched glass is a truly non-ergodic system. It will begin to relax towards the thermodynamic state which would have been typical upon slower quenching. But unlike at temperatures above vitrification, the structural re-arrangement below $T_{g}$ no longer depends only on the ambient temperature $T$ alone but also on the temperature history. The vibrational temperature is near ambient, while the (now mostly static) structure is representative of an equilibrated sample at a "fictive" temperature $T_{f}$. Strictly, one must detail the complete temperature schedule. Yet, since the relaxations near a glass transition on the routine laboratory scales are so slow - seconds and slower - that most quenches would result in a structure typical of a single temperature $T_{g}$, save the smaller vibrational amplitudes. This immediately implies the energy barrier distribution should be nearly temperature independent. If one further replaces the full barrier distribution by a single typical barrier, one expects the temperature dendence of relaxation times in frozen glasses to follow a simple Arrhenius law. A similar expectation serves as the basis of the phenomenological framework of Nayaranaswany-Moynihan-Tool:

$$
k_{n . e .}=k_{0} \exp \left\{-x_{N M T} \frac{\Delta E^{*}}{k_{B} T}-\left(1-x_{\mathrm{NMT}}\right) \frac{\Delta E^{*}}{k_{B} T_{f}}\right\} .
$$

where $E^{*}$ is the equilibrated apparent activation energy at $T_{g}$ and $x_{\mathrm{NMT}}$ lies between 0 and 1 . The general expression for activated transitions within RFOT theory in Eq.(14), which allows one to treat transitions from an arbitrary initial local state and implies the apparent activation energy below $T_{g}$ is significantly smaller than above the glass transition. The free energy difference $f_{\text {in }}-f_{\text {eq }}$ becomes nearly temperature independent below $T_{g}$, also consistent with the initial state energy being above the equilibrated value. On the other hand, the rate of the temperature change of the apparent activation energy above $T_{g}$ depends on the temperature dependence of the configurational entropy, see Eq.(16). The RFOT theory thus predicts the nonlinearity parameter $x_{\mathrm{NMT}}$ to be correlated with the fragility. A straightforward calculation yields the following simple relation [37]:

$$
m \simeq \frac{19}{x}
$$




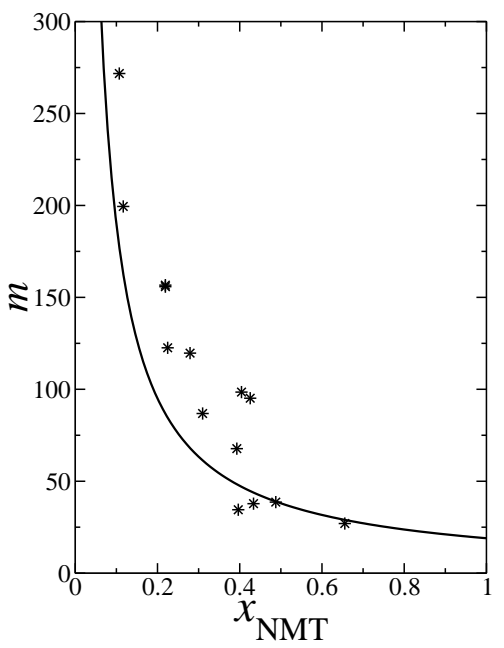

FIG. 14: The fragility parameter $m$ is plotted as a function of the NMT nonlinearity parameter $x_{\mathrm{NMT}}$. The curve is predicted by the RFOT theory when the temperature variation of $\gamma_{0}$ is neglected. The data are taken from Ref. [68]. The more fragile substances consistently lie above the prediction, which has no adjustable parameters. This discrepancy may be due to softening effects.

This prediction is compared to experimental data in Figure 14. (Here, we set $T_{f}=T_{g}$.) In addition to predicting the typical activation energy in frozen glasses, the RFOT theory can be employed to estimate the spread in the barrier distribution, and thus enable one to compute the temperature dependence of the exponent $\beta$ of the stretched exponential relaxation, as in Eq.(24). Corresponding experimental data addressing this prediction so far appear inconclusive [69, 70].

\section{QUANTUM THEORY OF GLASSES}

The microscopic picture of structural relaxations in supercooled liquids and glasses built up from the RFOT theory provides a starting point for understanding a radically different dynamical regime, i.e. the behavior of structural glasses at cryogenic temperatures [38], [39], [66], [71]. As we have recently provided a detailed account of these findings elsewhere [66, 71], we only summarize here several important points which help to strengthen the case for the RFOT theory predictions near $T_{g}$.

Much below the Debye temperature $T_{D}$, cryogenic glasses exhibit a set of excitations in excess of the usual Debye density of states. At temperatures below $1 \mathrm{~K}$ or so, these lead to a linear heat capacity and enhanced phonon scattering. In a higher temperature range around $10 \mathrm{~K}$ or so $(\sim 1 \mathrm{THz})$, yet another pronounced feature in the apparent density of excitations, called the "Boson Peak", is observed [72]. This excess density of states also rapidly shortens the mean free path of thermal phonons.

The mosaic predicted by RFOT theory immediately implies there are local structural excitations that may be viewed as local multi-level systems. These anharmonic motions would interact with the mostly elastic lattice. At low enough temperatures, one expects only the two lowest energy levels to contribute thermodynamically (but see below). This is in line with the early, phenomenological two-level system (TLS) model which empirically describes the low $\mathrm{T}$ regime $[73,74]$. But the low energy tail of the structural excitations can be computed from first principles. First one finds it is nearly energy independent at the lowest energies, as in the phenomenology. The corresponding density of states $\bar{P}$ is given by a simple expression [38]:

$$
\bar{P} \simeq \frac{1}{k_{B} T_{g} \xi^{3}}
$$

This gives a linear heat capacity whose magnitude is consistent with experiment. The condition of marginal stability of structural excitations to lattice distortions, at the glass transition, allows one to derive the coupling of those frozen-in transitions to the phonons:

$$
g \simeq \sqrt{k_{B} T_{g} \rho c_{s}^{2} a^{3}}
$$

where $\rho$ and $c_{s}^{2}$ are the mass density and the speed of sound of the glass. These two expressions, representing the nature of the states frozen at $T_{g}$, enable us to understand the mysterious universality of phonon scattering, found in many amorphous insulators: The ratio of the phonon mean free path to the phonon wave length depends on $\bar{P} g^{2}$ and turns out to be about 150 for all substances. Thus the standard tunneling model implies $\bar{P} g^{2} / \rho c_{s}^{2} \sim 10^{-2}[75]$. Combining Eqs.(29) and (30) from RFOT theory shows that the universality is expected because it reflects the nearly universal value of the cooperativity length at the glass transition [38]:

$$
\bar{P} g^{2} / \rho c_{s}^{2} \simeq\left(\frac{a}{\xi}\right)_{T_{g}}^{3} \simeq 1 / 200,
$$

Another simple correlation empirically found but otherwise unexpected follows from RFOT theory. It relates the TLS-phonon coupling $g$ to the glass transition temperature $T_{g}$ via the Lindemann ratio:

$$
g \simeq \frac{T_{g}}{\left(d_{L} / a\right)} \simeq 10 \cdot k_{B} T_{g}
$$

At the very lowest temperatures, the structural transitions involve the tunneling between two states of domain wall traversing a local region, spanning a length $\xi$ across. But the RFOT theory suggests these motions can support more than two states. At temperatures above $1 \mathrm{~K}$ or so, higher energy states of these tunneling centers are predicted to come into play. Microscopically, these motions can be visualized as vibrational excitations of the domain walls. The spectrum of these "capillary waves", 
or "ripplons", can be computed without adjustable parameters. The corresponding frequencies and density of states quantitatively account for the apparent excess heat capacity and phonon scattering in the terahertz range, that have been associated with the Boson Peak. The simplest quantitative estimate of the vibration frequencies is obtained neglecting interaction with the phonons and yields for the Boson Peak frequency [39]:

$$
\omega_{\mathrm{BP}} \simeq(a / \xi) \omega_{D},
$$

where $\omega_{D}$ is the Debye frequency. Effects of ripplonphonon interaction will lead to a shift and brodening of the ripplon resonances, and seem to explain the nonuniversality of the thermal conductivity plateau. The multilevel nature of "two level systems" in this temperature range is also apparent in single molecule spectroscopy experiments when complex spectral trails have ben observed by Orrit and coworkers [76].

The tunneling centers (TC) are fluctuating resonances and hence will mutually attract, following the usual London dispersion law $r^{-6}$. Since the number of active centers grows with temperature, this implies the inter-center attraction provides a mechanism for an additional attracting holding the solid together at higher temperatures. Like rubber, this yields a negative thermal expansion coefficient! Quantitative estimates [66] show that the attraction is greatly enhanced by the presence of ripplons, even at very low $T$. This mechanism yields a magnitude of the Grüneisen parameter consistent with experiment, in a number of glasses, which is often very anomalously large and negative [77].

Finally, individual molecular motions during the structural transitions will result in local electric charge redistribution. Ultimately this leads to a coupling of the transitions with electromagnetic waves. Recent estimates [71] show the transition induced electric dipole is about a Debye in magnitude, in spite of there being several hundred atoms participating in the motion. This value is consistent with experiments [78, 79].

\section{CONCLUDING REMARKS - FUTURE DIRECTIONS}

In place of a summary, we discuss the range of applicability of the RFOT theory; the limitations uncovered naturally suggest directions for future efforts.

We have already pointed out that the RFOT theory's predictions of the relaxation time and size scales are quantitative at viscosities of 10 Poise and above. This range of time scales constitutes, on the logarithmic scale, at least $80 \%$ of the routinely probed dynamic range in liquids of $10^{-2}$ to $10^{14}$ Poise or so. The ability of the theory to make predictions here relies on the fact that the motions are mesoscopic, hundreds of atoms being reconfigured, allowing sufficient thermodynamic averaging.

Based on mesoscale averaging the theory thus applies only when there is no significant medium range order.
Many liquids seem to satisfy this constraint, and span a wide range fragilities and chemical properties. These include nearly ionic compounds, such $\mathrm{SiO}_{2}, \mathrm{ZnCl}_{2}$; alcohols, such as propanol; aromatic and aliphatic hydrocarbons such as trinaphthylbenzene or 2-methylpentane. Measurements on such simple liquids confirm the predictions of the RFOT theory. The list of substances quantitatively described presently includes several dozens of materials and keeps growing.

On the other hand, some substances, while showing most of the signatures of glassy behaviors, exhibit additional types of order and scales of motion that will require separate treatment. We have in fact mentioned several examples of such systems: They include many of the most important polymers. In polymers, for instance, it is clear that various chain motions may provide long length scales and long time-scale relaxations, especially when the persistence length is large. Even for such systems, one still finds qualitative agreement with the RFOT calculations. For example, most polymers show TLS and Boson Peak densities of states that are comparable to those of simple molecular systems. One consequence of the possiblity of mid-range order is that one should exercise caution in searching for universalities in the glassy behaviours. The RFOT theory, among other things, provides in fact a first principles basis for such searches. As we have seen, substances that conform to the RFOT specifications, are predicted to show not one but rather a whole set of correlations, most of which we have mentioned earlier: $T_{K}$ vs. $T_{0}, \Delta c_{p}$ vs. $D$ (or $\left.m\right), \xi$ vs. $T, \xi\left(T_{g}\right)$ vs. $a$, universality of $s_{c}\left(T_{g}\right), \beta$ vs. $D, \beta$ vs. $T, m$ vs. $x, T_{c}$ vs. $\Delta c_{p}, \bar{P}$ vs. $T_{g}, \bar{P}$ vs. $\xi\left(\right.$ or $a$ ), $\bar{P}$ vs. $g, \omega_{\mathrm{BP}}$ vs. $\omega_{D}, \omega_{\mathrm{BP}}$ vs. $a$. The experiments become more difficult as one proceeds down the list, so fewer comparisons of predictions with experiment are available for the last few relations, but in our view the number of confirmed relationships already provides a secure basis to suggest anomalies are a sign of new degrees of freedom.

We were careful to limit the title of this review calling it "Theory of Structural Glasses and Supercooled Liquids", not dealing with all glassy systems. The quantitative applicability of RFOT theory to molecular glassy systems can be ultimately traced back to an emergent small parameter, the Lindemann ratio. Also for structural glasses, the glass transition temperature $T_{g}$ is much below the dynamic transition at $T_{A}$. This was pointed out clearly in an analysis by Eastwood and Wolynes [80] who developed a "Ginzburg" criterion for the theory. Their criterion shows many exciting systems exhibiting signs of glassiness are not in the appropriate universal regimes for strict quantitation because those systems are intrinsically softer with larger effective local motions. Colloidal glasses, owing to the larger size of their constituents, are intrinsically slow at the one particle level and thus at human measurement time scales, are always near $T_{A}$. Gels, stripe glasses [30], and microemulsions [81] have larger Lindemann ratios and although they are described by the RFOT theory at the mean-field level, again, we ex- 
pect there will be very significant renormalization of their behavior in the activated regime. Finally, although the RFOT theory provides a route from input intermolecular forces to the macroscopic behavior, the present quantitative successes of the theory avoid the hardest part of the detailed microscopic modeling by utilizing the fact that it is relatively easy to estimate configurational entropy from laboratory measurements. As usual, completely ab initio calculations will be more difficult, justifying greater attention to microscopic liquid state theory and molecular simulation technology.

Acknowledgments: V.L. has been funded in part by the GEAR Program and the New Faculty Grant at University of Houston. The work of P.G.W. is supported by the NSF grant CHE 0317017.
[1] J. D. van der Waals, Ph.D. thesis, Univ. Leiden (1873), english translation: Phys. Mem., vol. 1, p. 333, 1890.

[2] P. W. Anderson, Concepts in Solids (W. A. Benjamin, New York, 1963).

[3] J. Kepler, On the six-cornered snowflake (Clarendon Press, Oxford, 1966).

[4] P. Anderson, Science 267, 1615 (1995).

[5] C. A. Angell, J. Phys.: Condens. Matter 12, 6463 (2000).

[6] C. A. Angell, Proc. Natl. Acad. Sci. 92, 6675 (1995).

[7] J. D. Bernal, Proc. Roy. Soc. Ser. A 280, 299 (1958).

[8] R. Richert and C. A. Angell, J. Chem. Phys. 108, 9016 (1998).

[9] W. Kauzmann, Chem. Rev. 43, 219 (1948).

[10] L.-M. Martinez and C. A. Angell, Nature 410, 663 (2001).

[11] P. K. Dixon and S. R. Nagel, Phys. Rev. Lett. 61, 341 (1988).

[12] F. Mezei and M. Russina, J. Phys. Cond. Mat. 11, A341 (1999).

[13] J. R. Dorfman, T. R. Kirkpatrick, and J. V. Sengers, Ann. Rev. Phys. Chem. 45, 213 (1994)

[14] F. A. Lindemann, Phys. Z. 11, 609 (1910).

[15] Y. Singh, J. P. Stoessel, and P. G. Wolynes, Phys. Rev. Lett. 54, 1059 (1985).

[16] J. P. Stoessel and P. G. Wolynes, J. Chem. Phys. 80, 4502 (1984).

[17] T. V. Ramakrishnan and M. Yussouff, Phys. Rev. B 19, 2775 (1979).

[18] M. Yussouff, Phys. Rev. B 23, 5871 (1981).

[19] A. D. J. Haymet, Ann. Rev. Phys. Chem. 38, 89 (1987).

[20] J. J. Gilvarry, Phys. Rev. 102, 308 (1956).

[21] J. H. Bilgram, Phys. Rep. 153, 1 (1987).

[22] R. P. Gupta and P. K. Sharma, J. Chem. Phys. 48, 2451 (1968).

[23] T. R. Kirkpatrick and P. G. Wolynes, Phys. Rev. A 35, 3072 (1987).

[24] T. R. Kirkpatrick and D. Thirumalai, Phys. Rev. Lett. 58, 2091 (1987).

[25] T. R. Kirkpatrick and D. Thirumalai, Phys. Rev. B 36 , 5388 (1987).

[26] T. R. Kirkpatrick and P. G. Wolynes, Phys. Rev. B 36, 8552 (1987).

[27] M. Mézard and G. Parisi, Phys. Rev. Lett. 82, 747 (1999).

[28] B. Coluzzi, M. Mézard, G. Parisi, and P. Verrocchio, J. Chem. Phys. 111, 9039 (1999).

[29] S. Franz and J. Hertz, Phys. Rev. Lett. 74, 2114 (1995).

[30] J. Schmalian and P. G. Wolynes, Phys. Rev. Lett. 85, 3456 (2001).

[31] J.-P. Bouchaud and G. Biroli, J. Chem. Phys. 121, 7347 (2004).
[32] R. W. Hall and P. G. Wolynes, Phys. Rev. Lett. 90, 085505 (2003).

[33] H. S. Kang and F. H. Ree, J. Chem. Phys. 99, 2985 (1993).

[34] H. C. Andersen, J. D. Weeks, and D. Chandler, Phys. Rev. A 4, 1597 (1971).

[35] T. R. Kirkpatrick, D. Thirumalai, and P. G. Wolynes, Phys. Rev. A 40, 1045 (1989).

[36] X. Xia and P. G. Wolynes, Proc. Natl. Acad. Sci. 97, 2990 (2000).

[37] V. Lubchenko and P. G. Wolynes, J. Chem. Phys. 121, 2852 (2004).

[38] V. Lubchenko and P. G. Wolynes, Phys. Rev. Lett. 87, 195901 (2001).

[39] V. Lubchenko and P. G. Wolynes, Proc. Natl. Acad. Sci. 100, 1515 (2003).

[40] A. Saksaengwijit and A. Heuer, Phys. Rev. E 73, 061503 (2006).

[41] V. Lubchenko and P. G. Wolynes, J. Chem. Phys. 119, 9088 (2003).

[42] S. Franz, J. Stat. Phys. p. P04001 (2005).

[43] M. Dzero, J. Schmalian, and P. G. Wolynes, Phys. Rev. B 72, 100201(R) (2005).

[44] J. Villain, J. Physique 46, 1843 (1985).

[45] V. Lubchenko, unpublished.

[46] D. Turnbull, J. Appl. Phys. 21, 1022 (1950).

[47] G. Adam and J. H. Gibbs, J. Chem. Phys. 43, 139 (1965).

[48] V. Lubchenko (2006), to appear in J. Phys. Chem. B; cond-mat/0607009.

[49] V. N. Novikov, Y. Ding, and A. P. Sokolov, Phys. Rev. E 71, 061501 (2005).

[50] J. Stevenson and P. G. Wolynes, J. Phys. Chem. B 109, 15093 (2005).

[51] C. M. Roland, P. G. Santangelo, and K. L. Ngai, J. Chem. Phys. 111, 5593 (1999).

[52] U. Tracht, M. Wilhelm, A. Heuer, H. Feng, K. SchmidtRohr, and H. W. Spiess, Phys. Rev. Lett. 81, 2727 (1998).

[53] E. V. Russel and N. E. Israeloff, Nature 408, 695 (2000).

[54] S. F. Swallen, P. A. Bonvallet, R. J. McMahon, and M. D. Ediger, Phys. Rev. Lett. 90, 015901 (2003).

[55] X. Xia and P. G. Wolynes, J. Phys. Chem. 105, 6570 (2001).

[56] L. Berthier, G. Biroli, J. P. Bouchaud, L. Cipelletti, D. El Masri, D. L'Hote, F. Ladieu, and M. Perino, Science 310, 1797 (2005).

[57] X. Xia and P. G. Wolynes, Phys. Rev. Lett. 86, 5526 (2001).

[58] B. Böhmer, K. L. Ngai, C. A. Angell, and D. J. Plazek, J. Chem. Phys. 99, 4201 (1993).

[59] F. Stickel, E. W. Fischer, and R. Richert, J. Chem. Phys. 104, 2043 (1996). 
[60] J. D. Stevenson, J. Schmalian, and P. G. Wolynes, Nature Physics 2, 268 (2006).

[61] P. L. Leath, Phys. Rev. B 14, 5046 (1976).

[62] R. Hagedorn, Nuovo Cim. Suppl. 3, 147 (1965).

[63] V. N. Novikov and A. P. Sokolov, Phys. Rev. E 67, 031507 (2003).

[64] Y. Gebremichael, M. Vogel, and S. C. Glotzer, J. Chem. Phys. 120, 4415 (2004).

[65] V. Lubchenko (2005), to appear in J. Non.-Cryst. Sol.

[66] V. Lubchenko and P. G. Wolynes (2004), to appear in Adv. Chem. Phys.; cond-mat/0506708.

[67] J. Reinisch and A. Heuer, Phys. Rev. Lett. 95, 155502 (2005).

[68] C. A. Angell, K. L. Ngai, G. B. McKenna, P. F. Millan, and S. W. Martin, Appl. Phys. 88, 3113 (2000).

[69] A. Alegría, E. Guerrica-Echvarría, L. Goitiandía, I. Tellería, and J. Colmenero, Macromol. 28, 1516 (1995).

[70] R. L. Leheny and S. R. Nagel, Phys. Rev. B 57, 5154 (1998).

[71] V. Lubchenko, R. J. Silbey, and P. G. Wolynes, Mol. Phys. 104, 1325 (2006), cond-mat/0506735.
[72] W. A. Phillips, ed., Amorphous Solids: Low-Temperature Properties (Springer-Verlag, Berlin, Heidelberg, New York, 1981).

[73] P. W. Anderson, B. I. Halperin, and C. M. Varma, Philos. Mag. 25, 1 (1972).

[74] W. A. Phillips, J. Low Temp. Phys. 7, 351 (1972).

[75] J. J. Freeman and A. C. Anderson, Phys. Rev. B 34, 5684 (1986).

[76] A.-M. Boiron, P. Tamarat, B. Lounis, R. Brown, and M. Orrit, Chem. Phys. 247, 119 (1999).

[77] D. A. Ackerman, A. C. Anderson, E. J. Cotts, J. N. Dobbs, W. M. MacDonald, and F. J. Walker, Phys. Rev. B 29, 966 (1984).

[78] H. Maier, R. Wunderlich, D. Haarer, B. M. Kharlamov, and S. G. Kulikov, Phys. Rev. Lett. 74, 5252 (1995).

[79] B. M. Kharlamov, R. Wunderlich, H. Maier, and D. Haarer, J. Luminescence 76\&77, 283 (1998).

[80] M. P. Eastwood and P. G. Wolynes, Europhys. Lett. 60, 587 (2002).

[81] J. Schmalian and P. G. Wolynes, MRS Bull. 30, 433 (2005). 